\title{
Ergonomic Analysis of an Assembly Line Using the AnyBody Modeling System
}

\author{
Hilal Atici ${ }^{1}$, Demet Gonen ${ }^{2}$, Ali Oral ${ }^{3}$, Bunyamin Kaya ${ }^{4}$ \\ ${ }^{1}$ Department of Industrial Engineering, Faculty of Engineering, Uludag University \\ Gorukle Campus, Bursa, Turkey \\ hilalatici@balikesir.edu.tr \\ ${ }^{2}$ Department of Industrial Engineering, Faculty of Engineering, Balikesir University \\ Cagis Campus, Balikesir, Turkey \\ dgonen@balikesir.edu.tr \\ ${ }^{3}$ Department of Mechanical Engineering, Faculty of Engineering, Balikesir University \\ Cagis Campus, Balikesir, Turkey \\ alioral57@gmail.com \\ ${ }^{4}$ Nursan Wiring Harness R\&D Center \\ Organized Industrial Zone, 1. Street No: 5, Tavsanli, Kutahya, Turkey \\ bkaya@nursankd.com
}

\begin{abstract}
The employee performance as one of the most important factors affecting productivity and quality, is influenced by working methods and the conditions of working environment. Negative conditions in working environment cause decrease on employee performance, besides unsuitable working postures resulting from these conditions increase the risk of occupational musculoskeletal disorders. In this study, wiring harness assembly line of a company that produces wiring harnesses for the automotive industry was investigated. It was observed that during the assembly processes on the conveyor line, the bending and reaching movements of the employees are frequently repeated throughout the working hours. Employees work on a conveyor line with fixed height and slope angle. The purpose of this study is to improve working conditions and to protect employee health by reducing ergonomically unsuitable working postures. Firstly, the movements of the employees working in the assembly processes were analyzed using the Anybody Modeling System. According to the results of the analysis, it was seen that the bending and reaching movements had high values in muscle activations and joint reaction forces. As a result of the evaluation, the conveyor line was redesigned to be more compatible with employees. In the new assembly system designed; the height and inclination angle of assembly table can be adjusted according to the height of the employee to eliminate the reaching, turning or bending movements. Ergonomics analysis were repeated for the new conveyor line designed and it was seen that the risky and unhealthy postures of employees have decreased.
\end{abstract}

Keywords: Conveyor line, working posture, computer aided ergonomics analysis

\section{Introduction}

Unsuitable working postures that occur during work processes can cause pain and musculoskeletal disorders (MSD) in employees. MSD negatively affect the performance of employee and the quality of production. Examination and evaluation of working postures and taking preventive measures by determining the risks that may be caused by unsuitable working postures are important for the employee's health as well as work performance [1,2].

MSD of employees; occur due to repetition of physical movements such as bending, stretching and twisting that cause pain or complaints in muscles, nerves and some other soft tissues during work and exposure to these risk factors for a long time [3,4]. Disorganized layout of work environment, selection of unsuitable tools and equipment and inadequate break times are also effective in the formation of such movements that can create a risk [5].

In order to reduce the complaints of work related musculoskeletal disorders, improper postures on the assembly lines need to be corrected. Ergonomically correct and appropriate technical solutions ensure the reduction of strain in active muscles such as the waist, neck and leg [6,7]. Bernardes et al. (2012) aimed to reduce back pain in a shipping unit in their study. The layout of the assembly line was redesigned, some assembly tasks which pose a risk for back pain were removed and the employees were able to gain more control over their work [8]. 
Especially in cases of biomechanical overload and strain in assembly lines where labor intensive processes are mostly carried out, some changes in terms of ergonomics or redesign of the line are carried out in order to improve working postures. Computer aided analysis, virtual human models, virtual environment and simulations are useful for improving the assembly processes.

Ma et al. (2010) indicated that especially in assembly and maintenance works despite the use of automated techniques there are still too many manual transport operations and musculoskeletal disorders are one of the most important health problems due to physical overload and fatigue in these works. In this study; a new model was used to evaluate joint fatigue levels in manual operations, physical fatigue was defined and analyzes were performed using virtual human simulation techniques in the study [9]. Regazzoni and Rizzi (2014) also used virtual human models to simulate assembly and maintenance processes and to evaluate postures, stress and fatigue. They developed a motion capture method for obtaining human motion, transferred the data obtained from the motion capture to the simulation environment for the reproduction of human movements [10]. Battini et al. (2011) defined the relationship between assembly system design technics and ergonomics and developed a new theoretical structure for the design of assembly systems in connection with the ergonomic optimization in the workplace in their study [11].

There are also many studies in the automotive industry aimed at reducing musculoskeletal disorders in assembly processes. In the studies that mention new designs for this purpose [12,13]; analysis methods such as virtual human modeling, biomechanical analysis, posture analysis and employee interviews were used to assess the risk exposure of employees before and after the use of new design and the results showed that these applications help to prevent musculoskeletal disorders. Gonen et al. (2016) conducted computer aided ergonomics analysis to improve the unsuitable working postures in the assembly process of wheel hay rake which is one of the products of an enterprise that manufactures agricultural tools. According to the analysis results, the design of the assembly unit was changed and it is provided to be more ergonomic [14].

Different software can be used for ergonomic analysis. The AnyBody Modeling System was used for ergonomic analysis in this study. The AnyBody Modeling System (AMS) is a musculoskeletal modeling and biomechanical analysis software that uses the inverse dynamic principle to demonstrate the relationship between the human body and the working environment. Inverse dynamics means that the muscle force required to form a posture or movement given to the human model is calculated by the software [15]. The human model in AnyBody contains many of the muscles, joints and bones in the human body and the AMS is used to calculate the forces on the muscles, the joint reaction forces, the mechanical work and the efficiency for user specified dimensions and defined movements.

The human model was developed in the Anybody Modeling System and expressed in a total of 18 variable parameters such as spine flexion or extension, lateral flexion, pronation or supination of the right forearm [16]. Graichen et al. (2014) consider the manual assembly tasks in their study and constructed stress curves of the upper limb using the motion capture data and biomechanical body model of some selected assembly tasks [17].

\section{Case Study}

This study was conducted in a company that produces cable equipment for the automotive industry. The company is built on an area of $87.000 \mathrm{~m}^{2}$ with $27.000 \mathrm{~m}^{2}$ of indoor space. The product range of the company includes wiring harnesses that enable the electrical functions of passenger cars, light commercial and commercial vehicles to work. The wiring harness conveyor line where the production and assembly of the cable equipment is made is shown in Figure 1.

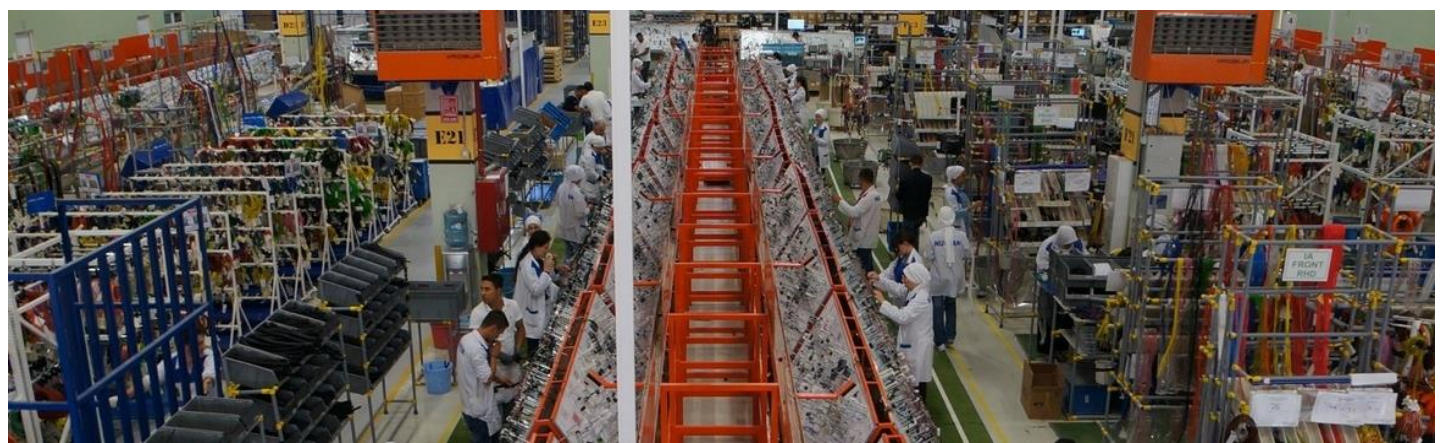

Fig. 1: Wiring harness conveyor line.

Operations performed on the conveyor line were examined in three phases: i) preliminary preparation, ii) cable laying and taping, iii) banding and accessory installation. In the preliminary preparation phase, some of the cables to be used in the equipment are plugged into the sockets in the grouping tables (Figure 2-a). During the cable laying phase, 
the cables prepared in the preliminary preparation phase are laid according to the indications on the assembly panel. There are eight different cable laying stations in conveyor line examined in this study. Each station has different cables that need to be installed. The work distributions of the stations are balanced and each employee concurrently completes the work in the station and goes to the other (Figure 2-b). In the banding and accessory installation phase, equipment is banded and accessories are installed according to the indications on the panel. In each station, employees' area to be taped is different (Figure 2-c).

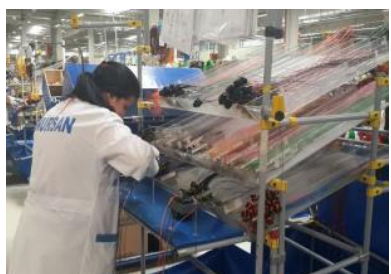

a) Preliminary preparation

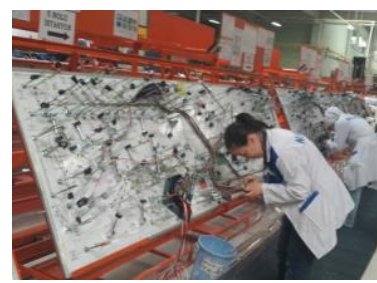

b) Cable laying and taping

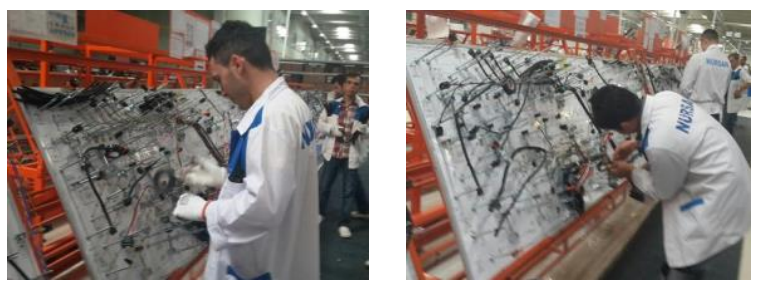

c) Banding and accessory installation

Fig. 1: Operations on the wiring harness conveyor line.

The main purpose of this study is to reduce ergonomically unsuitable working postures on the conveyor line and occupational diseases that can occur in employees. With the improvement studies to be done; it is aimed to express their importance for the company to the employees working on the conveyor line, to increase the productivity and work efficiency with the positive effect of employee satisfaction and also to decrease the treatment costs and the loss of labour due to the health problems that may arise in the employees. In this way, both employee and employer satisfaction will be increased.

In this study the data of human working posture is used as input in AMS. These data are body measurements of the employee and joint angles to define the body posture. AMS was used for analyzing the musculoskeletal system in order to quantitatively determine the unsuitable working postures and the biomechanical strain on the employees in the conveyor line.

\subsection{Analysis with the AnyBody Modeling System}

The working postures of employees were transferred to the AnyBody Modeling System and eight different working postures observed in the wiring harness conveyor line were analyzed. The maximum muscle activity (muscle effort in percent), the anteroposterior joint reaction force between the L4-L5 vertebrae and the glenohumeral anteroposterior joint reaction forces were considered as results of the analysis for each posture. In addition, the same analyzes were performed in the neutral posture of the AnyBody human model, so that the forces in the examined postures were compared with those in the neutral posture. Standing neutral posture refers to the motionless and unloaded posture that the head, neck and trunk are upright, chin is parallel to the floor, ears aligned with the shoulders from the side, in a straight $\mathrm{S}$ shape of the vertebrae, hands are beside the body with palms facing the body and the knees are flat and parallel to each other. The AnyBody human model in the neutral posture is given in Figure 3.

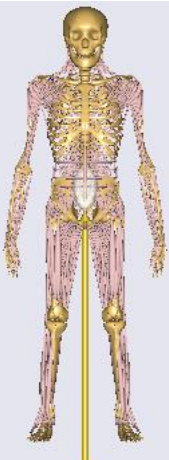

Fig. 3: AnyBody human model in neutral posture.

The muscle effort was about $14 \%$, left and right glenohumeral joint reaction forces were equal and $20.557 \mathrm{~N}$ and L4-L5 joint reaction force was $32.357 \mathrm{~N}$ as a result of analysis for the model in neutral posture with AMS. Images of working postures and virtual human models analyzed in the AnyBody Modeling System are shown in Figure 4-11. 

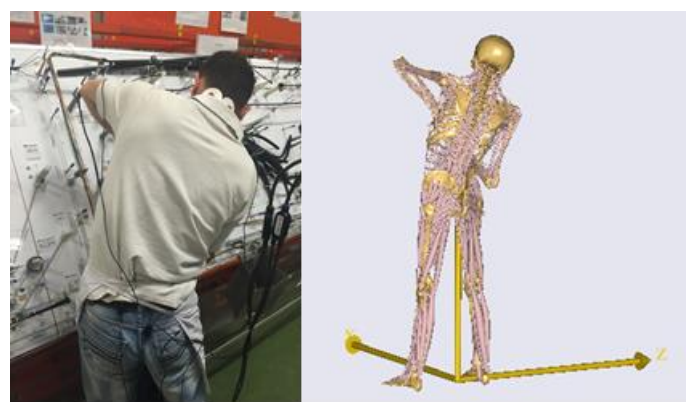

Fig. 4: AnyBody human model in working posture 1.
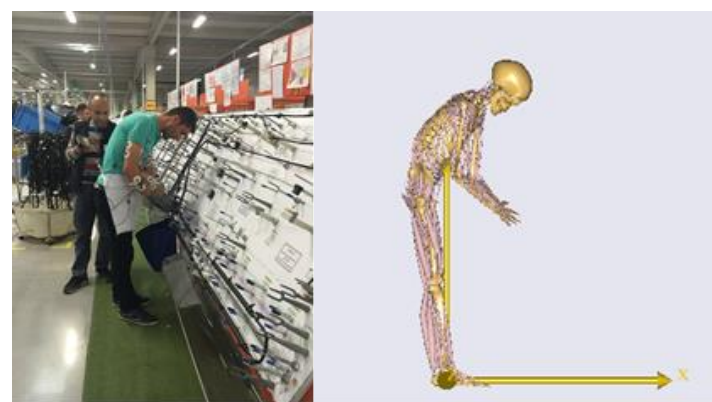

Fig. 5: AnyBody human model in working posture 2.

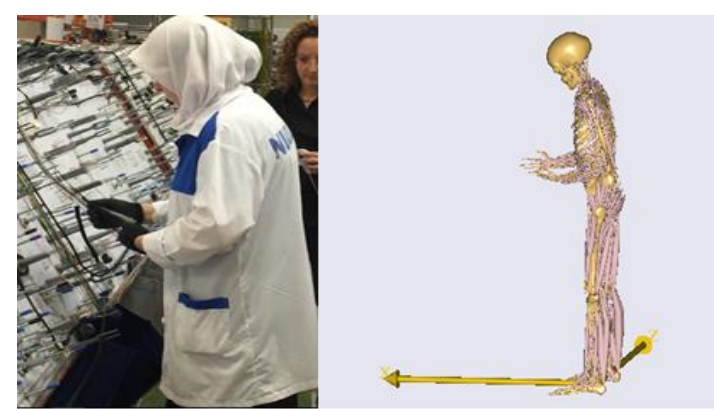

Fig. 6: AnyBody human model in working posture 3.
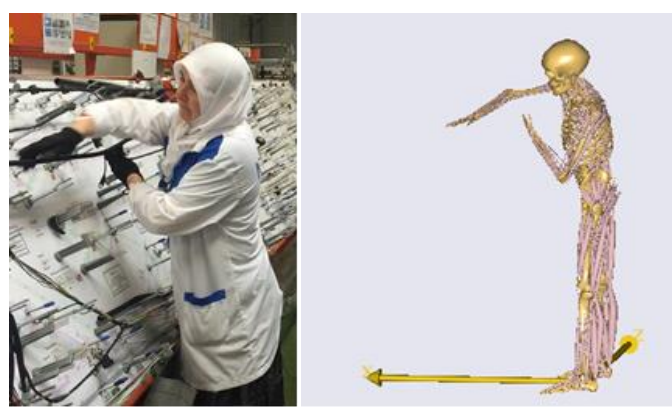

Fig. 7: AnyBody human model in working posture 4. 


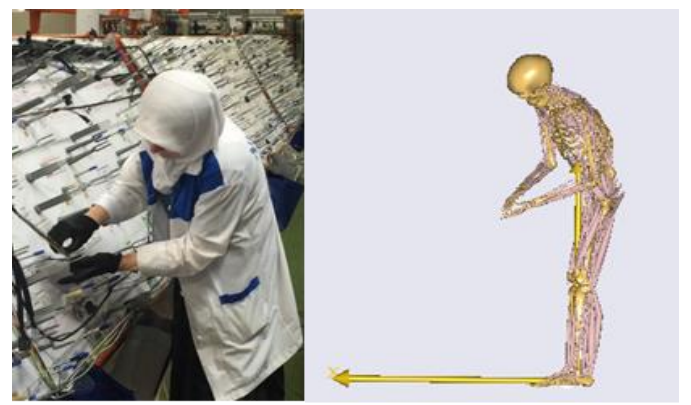

Fig. 8: AnyBody human model in working posture 5.

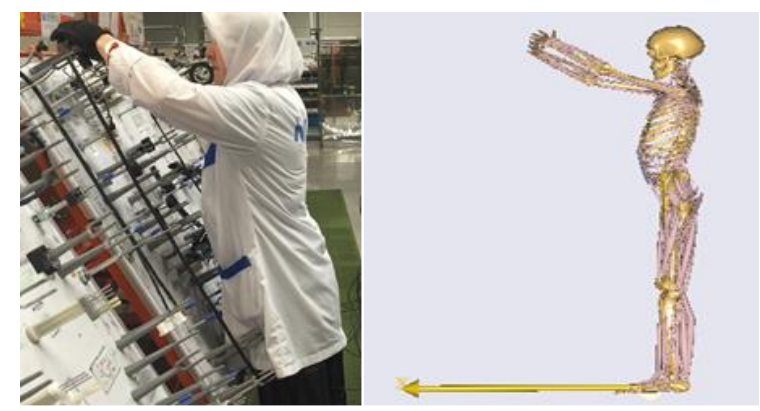

Fig. 9: AnyBody human model in working posture 6.
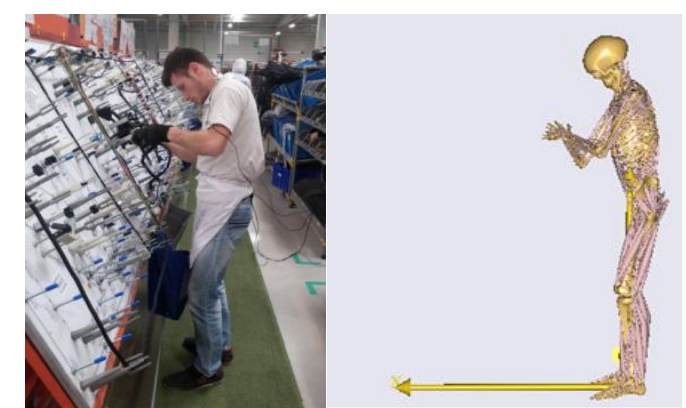

Fig.10: AnyBody human model in working posture 7.
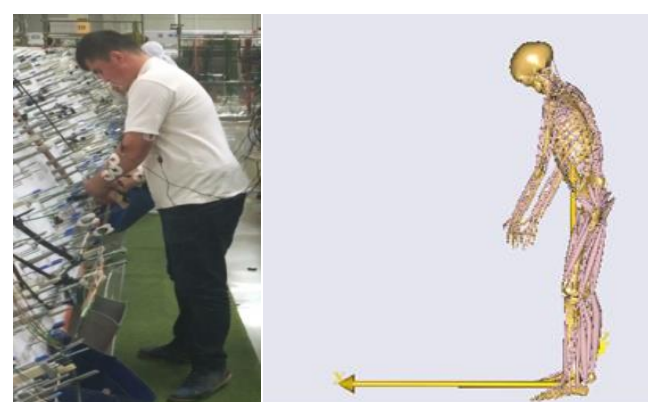

Fig. 11: AnyBody human model in working posture 8 .

The results of analysis made using AMS are given in Table 1. It was seen that maximum muscle activity was at most $52 \%$ at the $5^{\text {th }}$ working posture where the employee turns sideways and leans toward the bottom of the assembly table for cable tapping. Bending and rotation movements observed in the employees and similar difficulties that can cause back pain, mostly affect the joints between the $4^{\text {th }}$ and $5^{\text {th }}$ lumbar vertebrae. The highest joint reaction force occurred in the $2^{\text {nd }}$ working posture where the most flexion was seen among the employee and the lowest joint reaction force occurred in the $6^{\text {th }}$ working posture. In the $1^{\text {st }}$ working posture where the highest value of the joint reaction force of the left shoulder was observed, the employee's shoulder was raised up and rotated. The highest value of the joint reaction force of the right shoulder was seen in the $6^{\text {th }}$ working posture in which the employee reached up to work on the upper part of the assembly table. Considering the results obtained in the analysis, it was concluded that the high values belong to the working postures which force the employee biomechanically during the work day. 
Table 1: Maximum muscle activity, L4-L5 joint reaction force and shoulder joint reaction force values of working postures.

\begin{tabular}{|c|c|c|c|c|}
\hline Posture & $\begin{array}{c}\text { Maximum Muscle } \\
\text { Activity, Muscle } \\
\text { Effort (\%) }\end{array}$ & $\begin{array}{c}\text { L4-L5 Joint } \\
\text { Reaction Force (N) }\end{array}$ & $\begin{array}{c}\text { Left Glenohumeral } \\
\text { Joint Reaction Force } \\
\mathbf{( N )}\end{array}$ & $\begin{array}{c}\text { Right Glenohumeral } \\
\text { Joint Reaction Force } \\
\text { (N) }\end{array}$ \\
\hline neutral & 13.850 & 32.357 & 20.557 & 20.557 \\
\hline 1 & 46.376 & 87.402 & 144.013 & 5.168 \\
\hline 2 & 42.999 & 202.445 & 23.303 & 35.911 \\
\hline 3 & 20.351 & 72.835 & 15.504 & 1.429 \\
\hline 4 & 50.908 & 67.843 & 39.955 & 70.953 \\
\hline 5 & 52.837 & 143.995 & 8.876 & 6.667 \\
\hline 6 & 26.409 & 39.144 & 114.408 & 102.342 \\
\hline 7 & 30.411 & 98.138 & 87.091 & 49.463 \\
\hline 8 & 29.016 & 108.979 & 31.975 & 19.814 \\
\hline
\end{tabular}

In order to reduce ergonomically unsuitable working postures on the conveyor line and to prevent occupational diseases that may occur in employees, a conveyor line design was considered to reduce or even eliminate working postures that cause employees to suffer difficulties. The conveyor line design will enable the employees working on the conveyor line to work in more natural postures so that they can do their work efficiently and with less fatigue, thus avoiding work related complaints about musculoskeletal system.

\subsection{Redesign of the Wiring Harness Conveyor Line}

Since the anthropometric measurements of employees on wiring harness conveyor line with fixed height are different from each other, some improvements were considered for the conveyor moving at a certain height and slope. Redesign of the assembly line was carried out as the employee could work closest to the neutral working posture. In the new design, the assembly table will move according to the employee working on the relevant station when the conveyor is rotating. The mechanical design to provide the technical requirements was designed using SolidWorks software. The conveyor line design is shown in Figure 12.

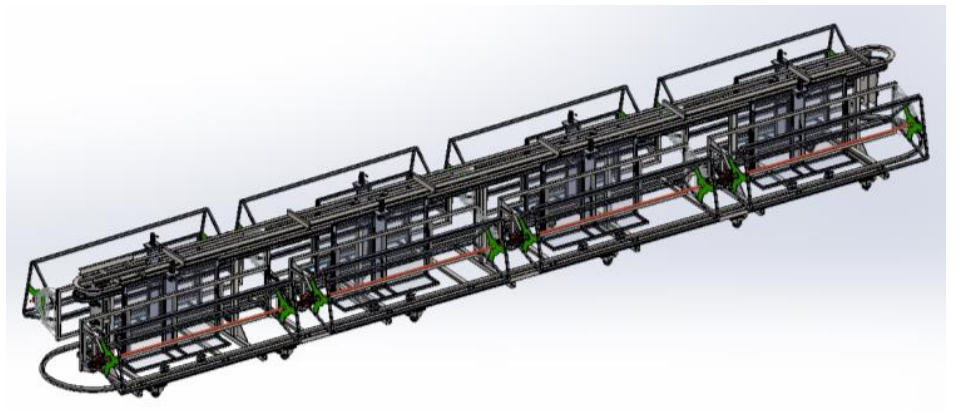

a) Conveyor line perspective view

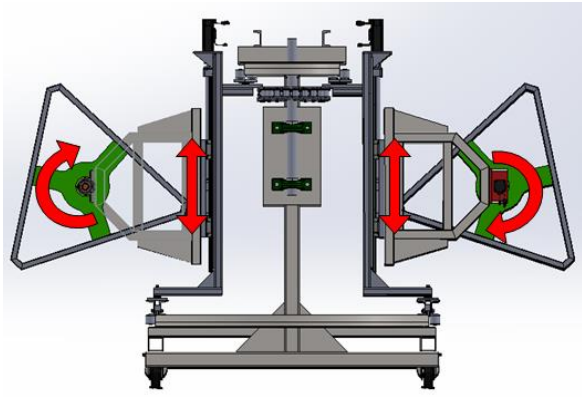

b) Conveyor line front view

Fig. 12: Conveyor line design.

The biggest difference in the new designed conveyor line compared to the existing conveyor is that the height and slope of the assembly tables are changeable. Thus, the height of the table will then be lowered or elevated according to the height of the employee working on the table and the employee will not need to reach up or lean down. In addition to this, the inclination of the table will change depending on the part of the employee is working on the table and there will be no need for the employee to reach or rotate. By adjusting the slope, the prongs on the table will also be prevented from disturbing the employee.

Movement of the assembly table according to the employee will be done with the help of barcodes and PLC system. Operator information will be placed in a barcode. The barcode system will be activated by reading the barcode of the operator with the barcode reader located at every table. Received barcode data will be transferred to the PLC system, servo motors installed in the assembly table will set the table according to the operator using the data coming to the PLC. 


\subsection{Analysis with the AnyBody Modeling System on Designed Conveyor Line}

The working postures of the employees in the wiring harness conveyor line were analyzed with AMS and the analysis were repeated for the new conveyor line designed considering all the investigation done. The AMS analysis results for the four working postures where the highest difficulties were observed in the analysis results are given in Table 2 in comparison with the current situation.

The highest muscle activity in the current conveyor line is expected to decrease from $52.8 \%$ in the $5^{\text {th }}$ posture to $19.1 \%$ in the designed conveyor line. The L4-L5 joint reaction force belongs to the working posture of the tall employee working downward in the present case and calculated to be $202.4 \mathrm{~N}$. It is predicted that this value will decrease to $73.4 \mathrm{~N}$ in the new design because the table height will be adjusted according to the height of the employee. The highest left shoulder joint reaction force $144.0 \mathrm{~N}$ which belongs to $1^{\text {st }}$ working posture is expected to reduce to $46.1 \mathrm{~N}$ and the highest right shoulder joint reaction force $102.3 \mathrm{~N}$ is expected to reduce to $20.5 \mathrm{~N}$.

Table 2: The results of AMS analysis for the working postures of existing and recommended situations of the conveyor line.

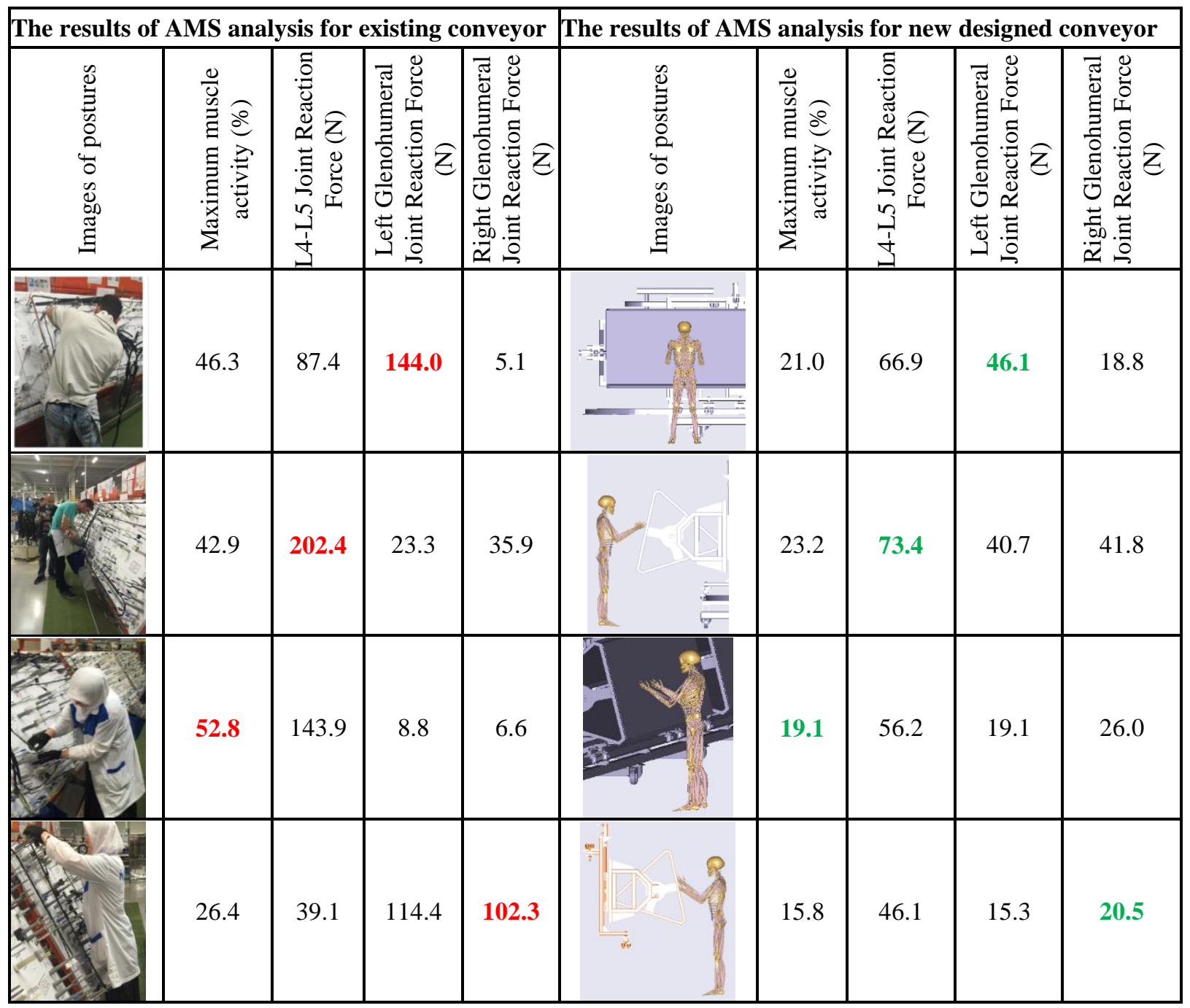

As a result of the AMS analysis for the designed conveyor line, muscle effort and joint reaction forces were generally lower than the current situation. Decrease in muscle effort means less fatigue in the employees, and decrease in joint reaction forces means less pain or discomfort in the waist and shoulders.

\section{Suggestions and Conclusion}

Ergonomically inadequate working postures in companies are those that can create risks and costs for both employers and employees. Costs incurred by work accidents or occupational diseases caused by working postures are undesirable costs for enterprises. For employees, there is a risk of musculoskeletal disorder due to the postures they are 
exposed to during the work processes. The mentioned working postures cause physiological difficulties that induce the employee's health to be adversely affected, psychologically force them and decrease the job performance.

In this study which is conducted in a company produces wiring harnesses of vehicles, the working postures observed on the wiring harness assembly line were ergonomically evaluated with the AnyBody Modeling System and the line was redesigned to reduce the musculoskeletal disorder risks identified. To make the conveyor line suitable for each employee, the conveyor assembly tables were redesigned so that they can move with variable height and inclination angle. The newly designed conveyor line was simulated in the virtual environment with AMS and the static analysis were repeated for the predicted working postures in the new conveyor. Muscle activities and joint reaction forces decreased according to the current situation. The most important feature of the new design is that the assembly tables can be moved according to the employee. With the table height and inclination becoming suitable for each employee, ergonomically undesirable postures will be avoided. This will provide a convenient and comfortable work environment for the employees. As a result, the protection of long term health and the increase of product quality and productivity will be ensured.

\section{References}

[1] D. Akay, M. Dagdeviren, M. Kurt, "Ergonomic analysis of working postures," Journal of the Faculty of Engineering and Architecture of Gazi University, vol. 18, no. 3, pp. 73-84, 2003.

[2] G. C. David, "Ergonomic methods for assessing exposure to risk factors for work-related musculoskeletal disorders," Occupational Medicine, vol. 55, pp. 190-199, 2005.

[3] B. P. Bernard, Musculoskeletal disorders and workplace factors: A critical review of epidemiologic evidence for work-related musculoskeletal disorders of the neck, upper extremity and low back pain. U.S. Department of Health and Human Services NIOSH Publication, 1997.

[4] I. L. Nunes, (2017, January 10). Introduction to musculoskeletal disorders, [Online]. Available: https://osha.europa.eu/en/topics/msds/index_html

[5] Anonym-5 (2017, January 10), Canadian Centre for Occupational Safety and Health (CCOHS), [Online]. Available: http://www.ccohs.ca/oshanswers/diseases/rmirsi.html

[6] B. Guner, S. Hasgul, "A study on physical disorders and risk factors observed in the assembly workers," 17 National Ergonomics Congress, Eskisehir, 2011.

[7] K. Swat, G. Krzychowicz, "ERGONOM: Computer-aided working posture analysis system for workplace designers," International Journal of Industrial Ergonomics, vol. 18, no. 1, pp. 15-26, 1996.

[8] J. M. Bernardes, C. Wanderck, A. Moro, "Participatory ergonomic intervention for prevention of low back pain: assembly line redesign case," Work: A Journal of Prevention, Assessment and Rehabilitation, vol. 41, pp. 59935998, 2012.

[9] L. Ma, D. Chablat, F. Bennis, W. Zhang, F. Guillauma, "A new muscle fatigue and recovery model and its ergonomics application in human simulation," Virtual and Physical Prototyping, vol. 5, no. 3, pp.123-137, 2010.

[10] D. Regazzoni, C. Rizzi, "Digital human models and virtual ergonomics to improve maintainability," ComputerAided Design and Applications, vol. 11, no. 1, pp. 10-19, 2014.

[11] D. Battini, M. Faccio, A. Persona, F. Sgarbossa, "New methodological framework to improve productivity and ergonomics in assembly system design," International Journal of Industrial Ergonomics, vol. 41, no. 1, pp. 3042, 2011.

[12] S. Ulin, W. Keyserling, "Case studies of ergonomic interventions in automotive parts distribution operations," Journal of Occupational Rehabilitation, vol. 14, no. 4, 307-326, 2004.

[13] A. Sundin, M. Christmansson, M. Larsson, "A different perspective in participatory ergonomics in product development improves assembly work in the automotive industry," International Journal of Industrial Ergonomics, vol. 33, no. 1, pp.1-14, 2004.

[14] D. Gonen, A. Oral, M. Yosunlukaya, "Computer-aided ergonomic analysis for assembly unit of an agricultural device," Human Factors and Ergonomics in Manufacturing \&Service Industries, vol. 26, no. 5, pp. 615-626, 2016.

[15] M. De Zee, L. Hansen, C. Wong, J. Rasmussen, E. Simonsen, "A generic detailed rigid-body lumbar spine model," Journal of Biomechanics, vol. 40, no. 6, pp.1219-1227, 2007.

[16] J. Rasmussen, C. Olesen, M. Lund, M. Andersen, S. Farahani, M. De Zee, "Prediction of motion in musculoskeletal models," ISB Technical Group on Computer Simaluation Symposium, Leuven, 2011.

[17] S. Graichen, T. Stein, B. Deml, "Adaptive workplace design based on biomechanical stress curves," Advances in Production Technology, pp.175-173, 2014. 